\title{
Shock compression modeling of metallic single crystals: comparison of finite difference, steady wave, and analytical solutions
}

\author{
Jeffrey T Lloyd ${ }^{1,3 *}$, John D Clayton ${ }^{1}$, Ryan A Austin ${ }^{2}$ and David L McDowell ${ }^{3,4}$
}

*Correspondence:

jeffrey.t.lloyd.civ@mail.mil

${ }^{3}$ Woodruff School

of Mechanical Engineering,

Georgia Institute

of Technology, Atlanta, GA

30332-0405, USA

Full list of author information

is available at the end of the

article

\begin{abstract}
Background: The shock response of metallic single crystals can be captured using a micro-mechanical description of the thermoelastic-viscoplastic material response; however, using a such a description within the context of traditional numerical methods may introduce a physical artifacts. Advantages and disadvantages of complex material descriptions, in particular the viscoplastic response, must be framed within approximations introduced by numerical methods.
\end{abstract}

Methods: Three methods of modeling the shock response of metallic single crystals are summarized: finite difference simulations, steady wave simulations, and algebraic solutions of the Rankine-Hugoniot jump conditions. For the former two numerical techniques, a dislocation density based framework describes the rate- and temperature-dependent shear strength on each slip system. For the latter analytical technique, a simple (two-parameter) rate- and temperature-independent linear hardening description is necessarily invoked to enable simultaneous solution of the governing equations. For all models, the same nonlinear thermoelastic energy potential incorporating elastic constants of up to order 3 is applied.

Results: Solutions are compared for plate impact of highly symmetric orientations (all three methods) and low symmetry orientations (numerical methods only) of aluminum single crystals shocked to $5 \mathrm{GPa}$ (weak shock regime) and $25 \mathrm{GPa}$ (overdriven regime).

Conclusions: For weak shocks, results of the two numerical methods are very similar, regardless of crystallographic orientation. For strong shocks, artificial viscosity affects the finite difference solution, and effects of transverse waves for the lower symmetry orientations not captured by the steady wave method become important. The analytical solution, which can only be applied to highly symmetric orientations, provides reasonable accuracy with regards to prediction of most variables in the final shocked state but, by construction, does not provide insight into the shock structure afforded by the numerical methods.

Keywords: Crystal plasticity, Finite difference method, Dislocations, Shock waves

\section{Introduction}

An understanding of the thermomechanical response of metallic crystals at high strain rates and high pressures is important for research and development of technologies involving impact, as occurring in crashworthiness applications and ballistic collisions,

(c) 2015 Lloyd et al.. This article is distributed under the terms of the Creative Commons Attribution 4.0 International License (http:// creativecommons.org/licenses/by/4.0/), which permits unrestricted use, distribution, and reproduction in any medium, provided you give appropriate credit to the original author(s) and the source, provide a link to the Creative Commons license, and indicate if changes were made. 
for example. Detailed constitutive models for single crystal thermoelastic-viscoplastic response enable prediction of effects of microstructure-e.g., lattice orientation, dislocation content, grain structure-on the performance of metals in such dynamic loading regimes. For modeling shocks of significant magnitude in single crystals, nonlinear elasticity, thermoelastic coupling, and material anisotropy become important. Models for the shock response of solids have witnessed continuous development and refinement since the mid-twentieth century [1-3], with theories involving various levels of detail, complexity, and efficiency available.

The finite difference (FD) approach to modeling shock wave propagation involves discretization of the solution domain in both space and time. Applications of FD methods towards descriptions of wave propagation in metals include [3-6]. Advantages of the method developed in Refs. [5, 6] include the following: crystals of any symmetry and orientation can be studied (i.e., transverse waves are captured), material properties may be heterogeneous in the (longitudinal) direction of wave propagation, and sophisticated rate- and temperature-dependent crystal plasticity models are enabled. Relative disadvantages are the time required for calculation of solutions and the need for artificial viscosity to regularize the shock width in the strong shock regime.

The steady wave (SW) approach to modeling shock waves presented in this work, which is strictly valid only for uniaxial strain conditions, involves transformation of governing partial differential equations to ordinary differential equations relative to a coordinate frame that moves along with a steady shock wave. Applications of the steady wave method towards descriptions of plastic shocks in metallic crystals include [7-11]. Advantages of the method developed in Ref. [10], which is the first known implementation of the SW approach for anisotropic elastic-plastic crystals, include the following: a detailed description of the steady shock structure (and associated material state) is obtained, solutions are obtained at relatively low computational cost, no artificial viscosity is used, and sophisticated rate- and temperature-dependent crystal plasticity models are enabled. Disadvantages are that effects of transverse waves for non-symmetric crystal orientations are ignored, unsteady waves cannot be addressed, and material properties must be spatially homogeneous.

The present analytical approach to modeling shocked metals involves simultaneous solution of the Rankine-Hugoniot jump conditions for conservation of mass, momentum, and energy, along with rate-independent constitutive equations for thermoelastic-plastic response. Previous work includes [12-15]. The present method, which can be applied only for symmetric crystal orientations (e.g., shocks propagating along [100] and [111] directions in FCC crystals), essentially reduces the problem to simultaneous solution of the yield condition and energy balance for the cumulative plastic slip and entropy, with the remaining conservation and constitutive laws sufficient for determination of the downstream material state. In this paper, "downstream" refers to material behind the plastic shock wave, "upstream" to material ahead of the shock. Advantages of this method are its simplicity (few material parameters are needed, and solutions are obtained nearly instantly) and ability to incorporate various nonlinear anisotropic thermoelastic potentials [16]. Disadvantages are the following: only highly symmetric orientations can be modeled as noted above, time dependence (e.g., explicit strain rate effects on strength) is ignored, and the shock is treated as a perfect jump discontinuity such 
that no further information regarding its structure (e.g., transitional values of state variables between upstream and downstream states) is obtained.

The remainder of this paper is outlined as follows. The FD model, the SW model, and the analytical model are described in "Finite difference model", "Steady wave model", and "Analytical model", including governing equations, constitutive theory and parameters, and numerical methods. Because these models have been described at length in prior publications $[6,10,15]$, only essential features are provided herein. Quantitative comparison and evaluation of the numerical approaches (FD and SW) are given in "Numerical methods comparison". Comparison of these results with the limited scope of results available from analytical solutions is given in "Comparison of numerical and analytical solutions". Concluding discussion follows in "Conclusion". The material of study is pure aluminum [ $\mathrm{Al}$, face centered cubic (FCC) structure], which is advantageous because of the extensive data available for its thermoelastic and shock response [17-19], and because it typically does not undergo twinning which would require more elaborate constitutive theory [20] than that employed herein.

Although all three models have been presented individually and validated versus experimental data in prior work $[6,10,15]$, previous papers have not included any comparisons of results among the three methods or any evaluations of computational efficiency. Explicit method comparisons identifying material orientations and loading regimes for which each method may be most appropriate are the primary new contributions of this paper. The only shocks considered herein are stable planar shocks as encountered in traditional plate impact experiments with null obliquity. Numerical methods developed to capture the behavior of converging and diverging shocks and their associated applications may be found elsewhere [21].

\section{Finite difference model}

The FD model evaluated in this paper incorporates constitutive theories for nonlinear anisotropic thermoelasticity and crystal plasticity described in detail in Ref. [6, 10]. Many, if not most, features are also used in the SW and analytical models described later in "Steady wave model" and "Analytical model".

Let $\nabla_{0}$ and $\nabla$ denote material and spatial gradients, respectively, and let $\boldsymbol{x}=\boldsymbol{x}(\boldsymbol{X}, t)$ denote spatial coordinates of a material point initially at $X$. The deformation gradient is decomposed into thermoelastic and plastic parts:

$$
\boldsymbol{F}=\nabla_{0} \boldsymbol{x}=\boldsymbol{F}^{E} \boldsymbol{F}^{P} .
$$

Let $\boldsymbol{v}=\dot{\boldsymbol{x}}$ be particle velocity. The velocity gradient is

$$
\boldsymbol{L}=\nabla \boldsymbol{v}=\dot{\boldsymbol{F}} \boldsymbol{F}^{-1}=\dot{\boldsymbol{F}}^{E} \boldsymbol{F}^{E-1}+\boldsymbol{F}^{E} \boldsymbol{L}^{P} \boldsymbol{F}^{E-1} .
$$

For adiabatic cases in the absence of discontinuities, local Lagrangian balances of mass, momentum, and energy are $[22,23]$

$$
\rho_{0}=\rho J, \quad \nabla_{0} \cdot \boldsymbol{P}=\rho_{0} \dot{\boldsymbol{v}}, \quad \dot{U}=\boldsymbol{P}: \dot{\boldsymbol{F}} .
$$

Here, $\rho_{0}$ and $\rho$ are initial and current mass densities, $J=J^{E} J^{P}=\operatorname{det} \boldsymbol{F}, \boldsymbol{P}$ is first PiolaKirchhoff stress related to symmetric Cauchy stress by $\boldsymbol{P}=J \boldsymbol{\sigma} \boldsymbol{F}^{-\mathrm{T}}$, and $U$ is internal energy per unit reference volume. 
The thermoelastic potential here depends on entropy per unit reference volume, $\eta$, and elastic Green strain, $\boldsymbol{E}^{E}$, the standard finite strain measure invoked in finite crystal plasticity theory $[5,10,24,25]$ :

$$
\boldsymbol{E}^{E}=\frac{1}{2}\left(\boldsymbol{F}^{E \mathrm{~T}} \boldsymbol{F}^{E}-\mathbf{1}\right)
$$

Other strain measures such as the Eulerian material strain $[6,16]$ and logarithmic strain $[15,26]$ have certain advantages for modeling large elastic compression; however, since the focus of the present work is comparison of methods of solution rather than constitutive theories, attention is restricted herein to the Green strain formulation. Letting Greek indices denote Voigt notation, internal energy is specified as

$$
U\left(\boldsymbol{E}^{E}, \eta\right)=\frac{1}{2} C_{\alpha \beta} E_{\alpha}^{E} E_{\beta}^{E}+\frac{1}{6} C_{\alpha \beta \delta} E_{\alpha}^{E} E_{\beta}^{E} E_{\delta}^{E}-\theta_{0}\left[\Gamma_{\alpha} E_{\alpha}^{E} \Delta \eta-f(\Delta \eta)\right] .
$$

Second- and third-order isentropic elastic constants are $C_{\alpha \beta}$ and $C_{\alpha \beta \delta}$; the Grüneisen tensor is $\Gamma_{\alpha}$; the reference temperature is $\theta_{0} ; \Delta \eta$ is entropy change from the reference state; and thermal energy is

$$
f=\exp \left(\Delta \eta / c_{0}\right)-1 \approx \Delta \eta+\frac{1}{2}(\Delta \eta)^{2} / c_{0},
$$

with $c_{0}$ the specific heat per unit volume at constant strain. Stored energy of defect substructure is omitted in (5) but could be incorporated following methods outlined in Refs. $[22,24,25]$. Such an assumption is considered reasonable for pure $\mathrm{Al}$, wherein experiments [27] indicate that over $90 \%$ of plastic work is dissipated as heat and contributes to temperature rise. Cauchy stress and temperature are given by

$$
\boldsymbol{\sigma}=J^{E-1} \boldsymbol{F}^{E}\left(\partial U / \partial \boldsymbol{E}^{E}\right) \boldsymbol{F}^{E \mathrm{~T}}, \quad \theta=\partial U / \partial \eta .
$$

Thermoelastic properties for aluminum are listed in Table 1 [6]. For crystals of cubic symmetry, $\Gamma_{I J}=\Gamma \delta_{I J}$, in indicial notation.

The plastic velocity gradient is, summing over slip systems $k$ with initial slip direction $\boldsymbol{s}^{k}$ and plane normal $\boldsymbol{m}^{k}$,

$$
\boldsymbol{L}^{P}=\dot{\boldsymbol{F}}^{P} \boldsymbol{F}^{P-1}=\sum_{k} b\left(N_{m}^{k} \bar{\nu}^{k}+\dot{N}_{h o m}^{k} \bar{x}\right) \boldsymbol{s}^{k} \otimes \boldsymbol{m}^{k} .
$$

Here, the magnitude of the Burgers vector is $b$, the mobile dislocation density is $N_{m}^{k}$ with glide velocity $\bar{v}^{k}$, and the rate of homogeneous nucleation is $\dot{N}_{\text {hom }}^{k}$ with a mean glide displacement $\bar{x}$. For FCC Al, slip occurs on up to $k=1,2, \ldots, 12\{111\}\langle 110\rangle$ systems, and

Table 1 Thermoelastic properties of Al $\left(\theta_{0}=300 \mathrm{~K}\right)$

\begin{tabular}{lll}
\hline Property & Value & Units \\
\hline$C_{11}, C_{12}, C_{44}$ & $106.7,60.4,28.3$ & $\mathrm{GPa}$ \\
$C_{111}, C_{112}, C_{123}$ & $-1,076,-315,36$ & $\mathrm{GPa}$ \\
$C_{144}, C_{155}, C_{456}$ & $-23,-340,30$ & $\mathrm{GPa}$ \\
$\Gamma$ & 2.30 & - \\
$C_{0}$ & 2.35 & $\mathrm{MPa} / \mathrm{K}$ \\
$\rho_{0}$ & 2.71 & $\mathrm{~g} / \mathrm{cm}^{3}$ \\
\hline
\end{tabular}


the corresponding ambient shear modulus is $\mu_{0}=C_{44}+\frac{1}{3}\left(C_{11}-C_{12}-2 C_{44}\right)$, leading to an initial shear wave velocity of $c_{s}=\sqrt{\mu_{0} / \rho_{0}}$; in the model, $c_{s}$ and $\mu$ are also updated with temperature and elastic strain [10]. The total dislocation density is $N^{k}=N_{m}^{k}+N_{i}^{k}$, where $N_{i}^{k}$ is the immobile density. Constitutive relations for the crystal-level mobile and immobile dislocation density evolution, as well as their associated mean velocity, build upon on previously developed isotropic constitutive models [3, 8, 9]. Letting $\tau^{k}=\boldsymbol{\sigma}:\left(\boldsymbol{F}^{E} \boldsymbol{s}^{k} \otimes \boldsymbol{m}^{k} \boldsymbol{F}^{E-1}\right)$ denote the resolved Cauchy stress on system $k$, evolution equations are [6]

$$
\begin{aligned}
\dot{N}_{m}^{k}= & \chi \dot{N}_{\text {hom }}^{k}+\dot{N}_{\text {het }}^{k}+\dot{N}_{m u l}^{k}-\dot{N}_{\text {ann }}^{k}-\dot{N}_{\text {tra }}^{k}, \\
\dot{N}_{i}^{k}= & (1-\chi) \dot{N}_{\text {hom }}^{k}+\dot{N}_{t r a}^{k} \\
\dot{N}_{\text {hom }}^{k}= & \dot{N}_{0} \exp \left[\left(g_{0 h o m} \mu b^{3} / k_{B} \theta\right)\left(\left|\tau^{k}\right| / \tau_{0 h o m}-1\right)\right], \\
\dot{N}_{h e t}^{k}= & \alpha_{h e t}\left|\dot{\tau}^{k}\right|(m+1)\left(\left|\tau^{k}\right|-\tau_{m i n}\right)^{m} /\left(\tau_{m a x}-\tau_{m i n}\right)^{1+m} \\
& \text { if } \tau_{m i n} \leq\left|\tau^{k}\right| \leq \tau_{m a x} \quad\left(\dot{N}_{h e t}^{k}=0 \text { otherwise }\right), \\
\dot{N}_{m u l}^{k}= & p_{m u l} N_{m}^{k} \sqrt{N_{f}^{k}}\left|\bar{v}^{k}\right|, \\
\dot{N}_{a n n}^{k}= & 2 \alpha_{a n n} b\left(N_{m}^{k}\right)^{2}\left|\bar{v}^{k}\right|, \\
\dot{N}_{\text {tra }}^{k}= & \alpha_{\text {tra }} N_{m}^{k} \sqrt{N_{f}^{k}}\left|\bar{v}^{k}\right| .
\end{aligned}
$$

Density rates corresponding to homogeneous nucleation, heterogeneous nucleation, multiplication, annihilation, and trapping are labeled by obvious subscripts. Densities of forest and parallel dislocations are, respectively,

$$
N_{f}^{k}=\sum_{l} N^{l}\left|\boldsymbol{m}^{k} \cdot\left(\boldsymbol{m}^{l} \times \boldsymbol{s}^{l}\right)\right|, \quad N_{p}^{k}=\sum_{l} N^{l}\left|\boldsymbol{m}^{k} \times\left(\boldsymbol{m}^{l} \times \boldsymbol{s}^{l}\right)\right| .
$$

Dislocation velocities are controlled by the following relations $[6,10]$ that involve physics of thermal activation at low stress and viscous drag at high stress:

$$
\begin{aligned}
& \bar{v}^{k}=\frac{c_{s} h^{k}}{\left[\exp \left(\Delta G^{k} / k_{B} \theta\right)-1\right]\left[c_{s} h^{k}\left(N_{f}^{k}\right)^{1 / 2} / \nu_{G}\right]+1} \\
& \text { if }\left|\tau^{k}\right|>\tau_{\text {pas }} \quad\left(\bar{v}^{k}=0 \text { otherwise }\right), \\
& \Delta G^{k}=\mu b^{3}\left\{1-\left[\left(\left|\tau^{k}\right|-\tau_{\text {pas }}^{k}\right) / \tau_{\text {cut }}^{k}\right]^{p}\right\}^{q},
\end{aligned}
$$




$$
\begin{aligned}
& \tau_{\text {pas }}^{k}=\alpha_{\text {pas }} \mu b \sqrt{N_{p}^{k}}, \quad \tau_{c u t}^{k}=\alpha_{c u t} \mu_{0} b \sqrt{N_{f}^{k}}, \\
& h^{k}=\left[\left(\xi^{k}\right)^{2}+1\right]^{1 / 2}-\xi^{k}, \quad \xi^{k}=B_{0} c_{s} /\left(2 \tau^{k} b\right) .
\end{aligned}
$$

Parameters entering (9)-(20) are compiled in Table 2; for a more thorough description of the dislocation density based framework for slip, see [6, 10, 22].

The present FD scheme permits particle displacements in all three Cartesian directions, but variations in displacement are permitted only in the direction of wave propagation denoted $X_{1}$, leading to the following matrix form of deformation gradient (1):

$$
[F]=\left[\begin{array}{lll}
\partial x_{1} / \partial X_{1} & 0 & 0 \\
\partial x_{2} / \partial X_{1} & 1 & 0 \\
\partial x_{3} / \partial X_{1} & 0 & 1
\end{array}\right]=\left[\begin{array}{lll}
F_{11} & 0 & 0 \\
F_{21} & 1 & 0 \\
F_{31} & 0 & 1
\end{array}\right]
$$

Balances of momentum and energy are often augmented with a scalar artificial viscosity $q$; correspondingly, from (2) and (3), for deformation of the form (21),

$$
\begin{aligned}
& \partial F_{i 1} / \partial t=\partial v_{i} / \partial X_{1}, \\
& \rho_{0}\left(\partial v_{i} / \partial t\right)=\partial P_{i 1} / \partial X_{1}-\left(\partial q / \partial X_{1}\right) \delta_{i 1}, \\
& \partial U / \partial t=\left(P_{i 1}-q \delta_{i 1}\right) \dot{F}_{i 1} .
\end{aligned}
$$

Letting subscripts followed by commas denote spatial discretization indices and superscripts denote temporal discretization indices, $\Delta t$ and $\Delta X_{1}$ the fixed time step and grid spacing, in discretized form (22)-(24) are

Table 2 Plastic properties of Al $\left(\theta_{0}=300 \mathrm{~K}\right)$

\begin{tabular}{llll}
\hline Property & Definition & Value & Units \\
\hline$b$ & Burgers vector & 0.286 & $\mathrm{~nm}$ \\
$N_{0}^{k}$ & Initial dislocation density & 0.56 & $1 / \mu \mathrm{m}^{2}$ \\
$f_{0}$ & Initial mobile disloc. fraction & 0.3 & - \\
$\dot{N}_{0}$ & Homogeneous gen. factor & $7.2 \times 10^{7}$ & $1 /\left(\mu \mathrm{m}^{2} \mu \mathrm{s}\right)$ \\
$g_{0 \text { hom }}$ & Homogeneous gen. parameter & 0.04125 & - \\
$\tau_{\text {ohom }} / \mu_{0}$ & Homogeneous gen. stress & 0.05 & - \\
$\chi$ & Mobile hom. disloc. fraction & 0.08 & - \\
$\bar{x} / b$ & Generation displacement & 13.3 & - \\
$\alpha_{\text {het }}$ & Heterogeneous gen. factor & 320 & $1 / \mu \mathrm{m}^{2}$ \\
$m$ & Heterogeneous gen. exponent & 0.8 & - \\
$\tau_{\text {min }} / \mu_{0}$ & Heterogeneous gen. bound & 0.004 & - \\
$\tau_{\text {max }} / \mu_{0}$ & Heterogeneous gen. bound & 0.04 & - \\
$p_{\text {mul }}$ & Multiplication probability & 0.088 & - \\
$\alpha_{\text {ann }}$ & Annihilation factor & 0.25 & - \\
$\alpha_{\text {tra }}$ & Trapping factor & 0.051 & - \\
$\alpha_{\text {pas }}$ & Passing strength factor & 0.1 & - \\
$\alpha_{\text {cut }}$ & Cutting strength factor & 0.9 & $1 / \mu \mathrm{s}$ \\
$\nu_{G}$ & Obstacle attempt frequency & $1 \times 10^{5}$ & - \\
$B_{0}$ & Drag coefficient & 18.0 & Pa $\mu \mathrm{s}$ \\
$p_{1} q$ & Strength exponents & $0.5,2$ & - \\
\hline & & &
\end{tabular}




$$
\begin{gathered}
\left(F_{i 1, i+1 / 2}^{n+1}-F_{i 1, i+1 / 2}^{n}\right) / \Delta t=\left(v_{i, i+1}^{n+1 / 2}-v_{i, i}^{n+1 / 2}\right) / \Delta X_{1}, \\
\frac{\rho_{0}}{\Delta t}\left(v_{i}^{n+1 / 2}-v_{i}^{n-1 / 2}\right)=\frac{1}{\Delta X_{1}}\left[\left(P_{i 1, i+1 / 2}^{n}-q_{i+1 / 2}^{n-1 / 2} \delta_{i 1}\right)-\left(P_{i 1, i-1 / 2}^{n}-q_{i-1 / 2}^{n-1 / 2} \delta_{i 1}\right)\right], \\
U_{i+1 / 2}^{n+1}-U_{i+1 / 2}^{n}=\left[\frac{1}{2}\left(P_{i 1, i+1 / 2}^{n+1}+P_{i 1, i+1 / 2}^{n}\right)-q_{i+1 / 2}^{n+1 / 2} \delta_{i 1}\right]\left[F_{i 1}^{n+1}-F_{i 1}^{n}\right] .
\end{gathered}
$$

Artificial viscosity (linear + quadratic) is computed as

$$
q_{i+1 / 2}^{n+1 / 2}=\frac{1}{2}\left(\rho_{i+1 / 2}^{n+1}+\rho_{i+1 / 2}^{n}\right)\left(a_{1} c_{l}\left|\Delta v_{1, i+1 / 2}^{n+1 / 2}\right|+a_{2}\left|\Delta v_{1, i+1 / 2}^{n+1 / 2}\right|^{2}\right)
$$

with $\Delta v_{1, i+1 / 2}^{n+1 / 2}=v_{1, i+1}^{n+1 / 2}-v_{1, i}^{n+1 / 2}, c_{l}$ the longitudinal linear elastic wave speed, $a_{1}=0.06$, and $a_{2}=2.0$. During expansion/rarefaction $\left(\rho_{i+1 / 2}^{n+1}-\rho_{i+1 / 2}^{n}<0\right), q=0$; note $q \geq 0$ follows from (28). The linear viscosity coefficient $a_{1}$ was chosen small enough to not influence the shock structure, whereas quadratic coefficient $a_{2}$ was chosen so that the shock would spread over three to five elements [28].

\section{Steady wave model}

The theory implemented in the current SW simulations $[10,11]$ is essentially equivalent to that of "Finite difference model", Eqs. (1)-(20) and properties in Tables 1 and 2. As discussed in detail in Ref. [10], the SW model invokes the Helmholtz free energy $\Psi$ as the fundamental thermodynamic potential, related to internal energy $U$ via the partial Legendre transformation

$$
\Psi=\Psi\left(\boldsymbol{E}^{E}, \theta\right)=U-\theta \eta
$$

Stress and entropy obey

$$
\boldsymbol{\sigma}=J^{E-1} \boldsymbol{F}^{E}\left(\partial \Psi / \partial \boldsymbol{E}^{E}\right) \boldsymbol{F}^{E \mathrm{~T}}, \quad \eta=-\partial \Psi / \partial \theta .
$$

Correspondences among properties in Table 1 and those entering a free energy function consistent with (5) (e.g., cubic in strain) are achieved via the usual Maxwell relations of nonlinear Lagrangian thermoelasticity [23, 29].

In contrast to (21), for the SW model it is assumed deformation is uniaxial and of the form below with $F_{11}=\lambda$ :

$$
[\boldsymbol{F}]=\left[\begin{array}{lll}
\partial x_{1} / \partial X_{1} & 0 & 0 \\
0 & 1 & 0 \\
0 & 0 & 1
\end{array}\right]=\left[\begin{array}{lll}
\lambda & 0 & 0 \\
0 & 1 & 0 \\
0 & 0 & 1
\end{array}\right]
$$

and is unable to model transverse waves captured by the FD method of "Finite difference model". However, also in contrast to the FD approach, no artificial viscosity is required for strong shocks, and the shock profile (e.g., width) is an outcome of the simulation 
rather than controlled by viscous regularization. Equations of continuity and momentum conservation reduce to

$$
\partial v_{1} / \partial X_{1}=\partial \lambda / \partial t, \quad \partial P_{11} / \partial X_{1}=\rho_{0}\left(\partial v_{1} / \partial t\right) .
$$

Introducing a coordinate $Y=X_{1}-D t$ moving with steady speed $D$ in the $\left(X_{1^{-}}\right)$direction of shock propagation, partial differential equations in (32) can be transformed to the ordinary differential equations

$$
\mathrm{d} v_{1} / \mathrm{d} Y=-D \mathrm{~d} \lambda / \mathrm{d} Y, \quad \mathrm{~d} P_{11} / \mathrm{d} Y=-\rho_{0} D\left(\mathrm{~d} v_{1} / \mathrm{d} Y\right) .
$$

Similarly, rate equations for plastic deformation, dissipative temperature rise, and dislocation densities become

$$
\begin{gathered}
\mathrm{d} \boldsymbol{F}^{P} / \mathrm{d} Y=-L^{P} \boldsymbol{F}^{P} / D, \quad \mathrm{~d}\left(\Delta \theta^{P}\right) / \mathrm{d} Y=-\dot{\theta}^{P} / D, \\
\mathrm{~d} N_{m}^{k} / \mathrm{d} Y=-\dot{N}_{m}^{k} / D, \quad \mathrm{~d} N_{i}^{k} / \mathrm{d} Y=-\dot{N}_{i}^{k} / D .
\end{gathered}
$$

Here, $\theta=\theta_{0}+\Delta \theta^{E}+\Delta \theta^{P}$, where $\Delta \theta^{P}$ results from plastic work and $\Delta \theta^{E}$ results from thermoelastic coupling (see [10] for full expressions). Integration of (33) from $+\infty$ gives the Raleigh line

$$
P_{11}-P_{11}^{+}=\rho_{0} D^{2}\left(\lambda-\lambda^{+}\right),
$$

where $(\cdot)^{+}$denotes a quantity evaluated at the beginning of the steady plastic wave; for a weak shock, this state corresponds to the elastic precursor and HEL; for a strong/ overdriven shock, $P_{11}^{+}=0$ and $\lambda^{+}=1$. In the numerical implementation, (34)-(36) are solved incrementally along the Raleigh line from the initial state $(\cdot)^{+}$to the end state $(\cdot)^{-}$ , where the latter is determined by the imposed boundary condition (e.g., shock stress $P_{11}^{-}$or volume ratio $V^{-} / V_{0}=\lambda^{-}$in the final shocked state at $\left.Y \rightarrow-\infty\right)$. The material response may be fully anisotropic, but the analysis ignores transverse waves that would arise from loading along crystal orientations with less than two-fold rotational symmetry [30] (neglecting nonlinear elastic effects from higher order elastic constants that may introduce longitudinal and transverse wave coupling [31]).

\section{Analytical model}

The present method of analytical solution, described more fully in Ref. [15], considers the Rankine-Hugoniot jump conditions for a steady planar shock [12]:

$$
\begin{aligned}
& \llbracket \rho \mathbf{v} \rrbracket=0, \quad \llbracket \sigma \rrbracket-\rho \mathbf{v} \llbracket \mathbf{v} \rrbracket=0, \\
& \llbracket \rho \mathbf{v}\left(u+\frac{1}{2} \mathbf{v}^{2}\right)-\sigma \mathbf{v} \rrbracket=0 .
\end{aligned}
$$

These conditions, which idealize the shock structure to infinitesimal thickness, effectively replace (3). The above conditions consider a continuous and initially homogeneous slab of material through which a planar shock moves, in the $X_{1}$-direction, with natural velocity $D$. As in "Steady wave model", let $(\cdot)^{+}$and $(\cdot)^{-}$label quantities in the material ahead (i.e., upstream) and behind (i.e., downstream) from the shock. Let $\llbracket(\cdot) \rrbracket=(\cdot)^{-}-(\cdot)^{+}$and $\langle(\cdot)\rangle=\frac{1}{2}\left[(\cdot)^{-}+(\cdot)^{+}\right]$denote the jump and average of a quantity across the shock. Let $\boldsymbol{n}$ be a unit normal vector to the planar shock, i.e., $\boldsymbol{n}=\partial \boldsymbol{x} / \partial x_{1}$. The only nonvanishing 
component of particle velocity is $v=\boldsymbol{v} \cdot \boldsymbol{n}$. The Cauchy stress component normal to the shock front is $\sigma=\boldsymbol{\sigma}:(\boldsymbol{n} \otimes \boldsymbol{n})=\sigma_{11}$. The relative velocity of the material with respect to the shock is $v=v-D$. Internal energy per unit mass is $u=U / \rho_{0}$. Using (37), (38) can be rewritten as [12]

$$
\llbracket u \rrbracket=\langle\sigma\rangle \llbracket 1 / \rho \rrbracket \Leftrightarrow \llbracket U \rrbracket=\langle\sigma\rangle \llbracket \rrbracket .
$$

The downstream state is defined by the set of variables $\left(v^{-}, \rho^{-}, \sigma^{-}, u^{-}\right)$. The RankineHugoniot conditions give three equations for determining this state; in order to fully define the downstream state, a fourth equation is supplied by the constitutive model. Here, the constitutive theory for thermoelastic response is identical to the anisotropic nonlinear Lagrangian theory of "Finite difference model", Eqs. (1), (4)-(7), and Table 1. Considered are longitudinal elastic-plastic shocks corresponding to planar impact in pure mode directions in single crystals (i.e., directions parallel to an axis of two-fold or greater rotational symmetry). A sample of material subjected to a step or ramp loading in normal stress, with no applied shear stress, develops a two-wave structure consisting of a single longitudinal elastic wave (i.e., the elastic precursor), followed by a single longitudinal plastic wave of velocity $D$ if the HEL is exceeded. For overdriven shocks, there is no precursor. Total deformation is

$$
\begin{aligned}
& {\left[\boldsymbol{F}^{+}\right]=\left[\begin{array}{rrr}
\lambda^{+} & 0 & 0 \\
0 & 1 & 0 \\
0 & 0 & 1
\end{array}\right]=\left[\boldsymbol{F}^{E+}\right],} \\
& {\left[\boldsymbol{F}^{-}\right]=\left[\begin{array}{rrr}
\lambda^{-} & 0 & 0 \\
0 & 1 & 0 \\
0 & 0 & 1
\end{array}\right]=\left[\boldsymbol{F}^{E-} \boldsymbol{F}^{P-}\right] .}
\end{aligned}
$$

For the highly symmetric [100] orientation considered in "Comparison of numerical and analytical solutions", $n=8$ glide systems are active simultaneously at shock stresses exceeding the HEL stress $P_{H}=-\sigma^{+}=-P_{11}^{+}$, all at the same rate [13]. For monotonic loading, integration of (8) yields the plastic deformation:

$$
\begin{aligned}
\boldsymbol{F}^{\mathrm{P}}(\gamma)= & \exp \left(\gamma \sum_{k} \boldsymbol{s}^{k} \otimes \boldsymbol{m}^{k}\right) \\
& \approx \mathbf{1}+\gamma \sum_{k} \boldsymbol{s}^{k} \otimes \boldsymbol{m}^{k}+\frac{\gamma^{2}}{2}\left(\sum_{k} \boldsymbol{s}^{k} \otimes \boldsymbol{m}^{k}\right)^{2}+\frac{\gamma^{3}}{6}\left(\sum_{k} \boldsymbol{s}^{k} \otimes \boldsymbol{m}^{k}\right)^{3}
\end{aligned}
$$

with cumulative shear $\gamma$, which accounts for slip and nucleation contributions, to be determined as an outcome of the analysis. The exponential solution in the first of (42) is exact when the plastic shearing rate is constant, i.e., when $\dot{\gamma}=\gamma / t=$ constant. The series approximation in the second of (42) is accurate to third order in shear and was sufficient for problems considered here, where the maximum values of $\gamma$ are on the order of 0.1. From the geometry of the problem, all $n$ systems experience the same resolved shear stress $\tau=\tau^{k}$. In lieu of the viscoplastic model implemented in "Finite difference model" and "Steady wave model", for the analytical treatment a two-parameter yield criterion in the plastically deforming regime is prescribed: 


$$
J \tau / \mu_{0}=g(\gamma) / \mu_{0}=g_{0}+H n \gamma .
$$

Here, $g_{0}$ is dimensionless initial shear strength at the HEL, dependence of strength $g=g^{k}$ on temperature is omitted, and hardening is proportional via constant $H$ to the total slip on all $n$ active systems. The factor of $J=J^{E}$ in (43) accounts for work conjugacy of Kirchhoff stress and plastic slip in the intermediate configuration implied by the multiplicative decomposition of $\boldsymbol{F}$ in (1) [23].

Assume that HEL shock stress $P_{H}$ is known from experiment. Then the upstream (HEL) state is fully determined by the analytical solution in Refs. [16, 29]. Specifically, $\lambda$ is decreased incrementally until $P=-P_{11}=-\sigma_{11}$ reaches $P_{H}$ (positive in compression), at which point $\lambda=\lambda^{+}$and $U=U^{+}$. Given total deformation $\lambda^{-}$and slip variable $\gamma$, thermoelastic deformation behind the plastic shock is known from $\boldsymbol{F}^{E}=\boldsymbol{F}\left(\lambda^{-}\right) \boldsymbol{F}^{P-1}(\gamma)$ . Internal energy, axial shock stress, and shear stress can then be written as

$$
U^{-}=U^{-}\left(\lambda^{-}, \gamma, \eta^{-}\right), \quad P^{-}=P^{-}\left(\lambda^{-}, \gamma, \eta^{-}\right), \quad \tau=\tau\left(\lambda^{-}, \gamma, \eta^{-}\right) .
$$

Note that the full thermoelastic constitutive model is required for evaluation of (44). Let $\lambda^{-}=V^{-} / V_{0}$ be prescribed as the load parameter. Then energy balance (39) and yield criterion (43) comprise two coupled algebraic equations that can be solved simultaneously for $\gamma$ and $\eta^{-}$:

$$
\begin{aligned}
& U^{-}\left(\lambda^{-}, \gamma, \eta^{-}\right)-U^{+}=\frac{1}{2}\left[P^{-}\left(F^{-}, \gamma, \eta^{-}\right)+P_{H}\right]\left[\lambda^{+}-\lambda^{-}\right], \\
& \tau\left(\lambda^{-}, \gamma, \eta^{-}\right) / \mu_{0}=\left(g_{0}+H n \gamma\right) / \lambda^{-} .
\end{aligned}
$$

To obtain Hugoniot stress versus volume curves, (46) and (45) are solved simultaneously for $\gamma$ and $\eta^{-}$as $\lambda^{-}$is decreased incrementally from the HEL state. With shock stress computed from the second of (44), plastic shock velocity $D$ and downstream particle velocity $v^{-}$can be obtained from the Hugoniot equations for mass and momentum conservation in (37), leading to [7]

$$
D=\left\{\left(P^{-}-P_{H}\right) /\left[\rho_{0}\left(\lambda^{+}-\lambda^{-}\right)\right]\right\}^{1 / 2}, \quad v^{-}=v^{+}-D\left(\lambda^{-}-\lambda^{+}\right) .
$$

The downstream state is now fully known. For aluminum single crystals, the first strength parameter $g_{0}=7.2 \times 10^{-4}$ is known from the nonlinear elastic solution [16] at $P_{H}=0.1 \mathrm{GPa}$ [10], corresponding to $\lambda^{+} \approx 0.999$, and thus does not require calibration. The second parameter, hardening constant $H=0.05$, is calibrated such that cumulative plastic deformation predicted by the analysis for shocks in the regime $P_{H} \leq P^{-} \leq 25$ GPa is in respectable agreement with that predicted by the numerical methods of "Finite difference model" and "Steady wave model", which have been compared extensively to experiments $[6,10]$. The calibration of $H$ will be explained later in "Comparison of numerical and analytical solutions". Much of the foregoing discussion applies for weak shocks; for strong shocks, conditions $P_{H} \rightarrow P^{+}=0, \lambda^{+}=1, v^{+}=0$, and $U^{+}=0$ are enforced.

\section{Numerical methods comparison}

Approximations introduced by the FD and SW numerical methods are evaluated quantitatively via examination of results of four representative test problems, whose shock 
Table 3 Numerical simulations and approximations

\begin{tabular}{lll}
\hline Simulation & SW approximations & FD approximations \\
\hline $5 \mathrm{GPa}[100]$ & None & None \\
$5 \mathrm{GPa}$ low symmetry & Uniaxial $\boldsymbol{F}$ & None \\
$25 \mathrm{GPa}[100]$ & None & Artificial viscosity \\
$25 \mathrm{GPa}$ low symmetry & Uniaxial $\boldsymbol{F}$ & Artificial viscosity \\
\hline
\end{tabular}

strengths are given in Table 3. Because the SW method approximates strain as uniaxial, it is expected to give highly accurate solutions for impact problems wherein the target is shocked along a direction that possesses twofold or greater rotational symmetry (which results in uniaxial strain), and give approximate solutions for problems of lower symmetry (in which quasi-longitudinal and quasi-transverse may form). The FD method is able to model weak shock loading problems without an artificial viscosity given a mesh resolution that can sufficiently resolve the shock width; however, in the strong shock regime viscous regularization is used to damp the large jump in velocity that precedes plastic deformation [6]. The approximations associated with each of the four problems are summarized in Table 3. In low symmetry simulations, the crystal is rotated from the reference frame via Bunge angles $\phi_{1}=43.7^{\circ}, \Phi=49.26^{\circ}$, and $\phi_{2}=132.8^{\circ}$, producing the orientation in Figure 1. The FD mesh resolution $\left(\Delta X_{1}\right)$ is fixed, and a consistent step size is chosen for SW simulations so that approximately the same number of points is used to resolve the $\mathrm{SW}$ in all simulations.

\section{Velocity profiles}

In FD simulations at $P^{-}=5 \mathrm{GPa}$, the shock was generated by longitudinal plate impact of an a-sapphire impactor (X-cut). In all FD simulations, the a-sapphire impactor was modeled using isentropic thermoelasticity with an internal energy potential third order in elastic Green strain and elastic constants from Ref. [32]. The shock response was sampled from an interior point located $5.0 \mathrm{~mm}$ from the impact surface for these simulations, which were discretized using a mesh resolution of $\Delta X_{1}=0.83 \mu \mathrm{m}$. Velocity profiles from the SW and FD simulations of [100] and low symmetry orientations are compared in Figure 2. Because SW simulations are based on relative time, the wave profile is adjusted so that it is centered on the FD solution, which uses total time after impact.

For the [100] orientation, Figure 2a indicates that the SW and FD simulations give nearly identical results. This agreement is expected as neither method introduces

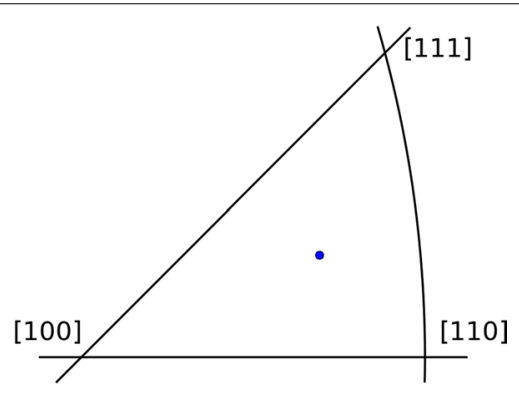

Figure 1 Inverse pole figure of low symmetry orientation. 

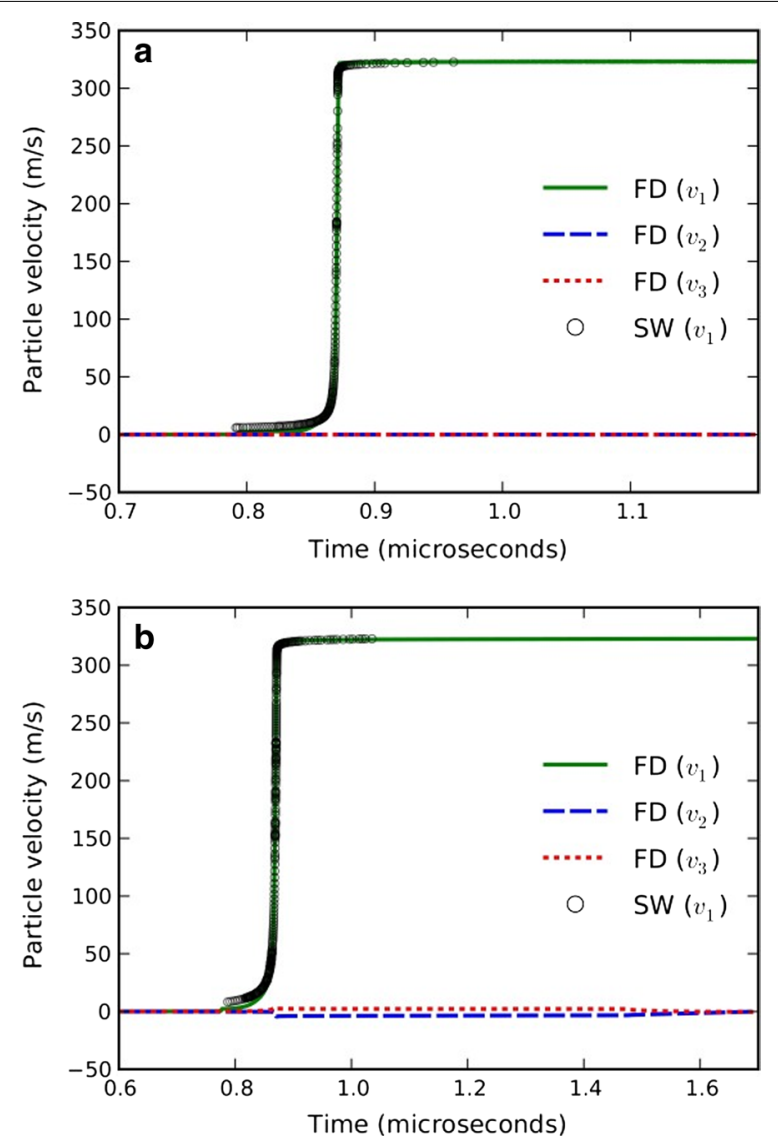

Figure 2 Velocity profiles computed using SW and FD methods ( $P^{-}=5 \mathrm{GPa}$ ) for a single crystal with $\mathbf{a}$ [100] orientation and $\mathbf{b}$ low symmetry orientation.

intrinsic approximations for this orientation and shock strength (Table 3). For the low symmetry orientation, Figure $2 \mathrm{~b}$ shows that although the SW method approximates deformation as uniaxial, it predicts a nearly identical longitudinal component of the velocity profile as the FD simulation. Although the wave profiles are nearly identical, the SW method under-predicts the peak resolved shear stress that occurs on slip systems by approximately $10 \%$ because it does not include the shear components of the quasi-longitudinal wave [22]. However, this appears to negligibly influence the longitudinal component of the velocity profile at the low impact stress.

In FD simulations at $P^{-}=25 \mathrm{GPa}$, the shock was again generated by longitudinal plate impact via an a-sapphire impactor; however, in this case an artificial viscosity was used. The shock response was sampled from an interior point located $20.0 \mu \mathrm{m}$ from the impact surface for these simulations, which were discretized using a mesh resolution of $\Delta X_{1}=5.0 \mathrm{~nm}$. Velocity profiles from the SW and FD simulations of [100] and low symmetry orientations are compared in Figure 3.

For the [100] orientation, wave profiles for the two methods are shown in Figure 3a. Because the SW method begins to track the solution at an adiabatic elastic compression for which the longitudinal elastic wave speed equals the SW speed (in this case, at a particle velocity of approximately $0.55 \mathrm{~km} / \mathrm{s}$ ), it gives no additional information concerning 

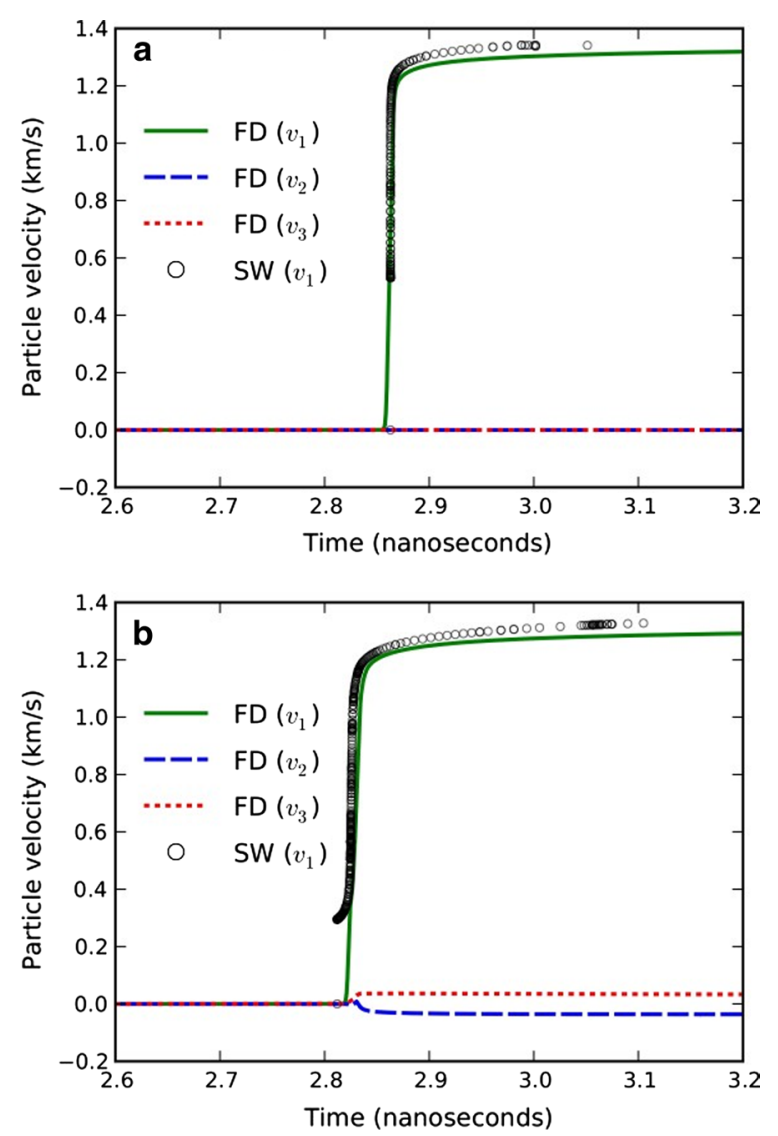

Figure 3 Velocity profiles computed using SW and FD methods ( $P^{-}=25 \mathrm{GPa}$ ) for a single crystal with a [100] orientation and $\mathbf{b}$ low symmetry orientation.

the wave profile up to this velocity; however, any wave structure calculated by the FD method up to this velocity is due to the artificial viscosity, so no physical insight is gained in FD simulations up to this velocity either. Above this velocity, the SW method predicts a slightly sharper rise than the FD method. This is because even though an extremely fine mesh resolution is employed there is still a smearing effect from the artificial viscosity, which decreases the peak strain rate experienced in the material. This decrease in peak strain rate decreases the rate of homogeneous dislocation by two orders of magnitude, which in turn alters the wave profile at elevated velocities, as fewer mobile dislocations are available to relax the deviatoric response through glide. Even though the coupling between viscoplasticity and viscous regularization is undesirable, the FD and SW methods predict nearly identical strength and accumulated plastic strain, although their wave profiles differ slightly.

Computed wave profiles for the low symmetry orientation in Figure 3b differ in several respects. The FD method captures transverse components of formation of the quasi-longitudinal wave which cannot be considered using the SW method. Additionally, the FD method predicts a single wave structure, whereas the SW method indicates deformation preceding the main rise. As discussed previously, local adiabatic treatment of overdriven shocks provides no information until the point where the elastic wave speed equals the 
SW speed. However, for the low symmetry shock, since SW simulation (longitudinal wave) has a different elastic stiffness than the FD simulation (quasi-longitudinal wave), the point where viscoplastic deformation occurs differs. This causes the two methods to predict differing shock structures, where the SW method predicts a dual shock structure and the FD method predicts an overdriven shock. Additionally, the FD method experiences elevated shear stresses due to the transverse deformation components, which gives a slightly different viscoplastic response as well. Based on these observations, the SW method appears unsuitable for simulations in which transverse wave components have a relatively large magnitude, i.e., simulation of strong shocks in single crystals with low symmetry orientations.

\section{Computational efficiency}

Because the SW method converts governing partial differential equations in space-time to ordinary differential equations in a steadily moving coordinate frame, it is expected to be significantly more computationally efficient than the FD method. This assertion is verified by computation times for simulations described in "Velocity profiles". Table 4 shows that the SW method is $\approx 2,000-8,000 \times$ faster than the FD method.

For the FD simulations presented in this work, one reason that the total computation times are several orders of magnitude longer than SW simulations is that a sufficiently fine mesh is employed so that dissipation is primarily due to the viscoplastic constitutive equation and not from artificial viscous regularization. To illustrate the effect of mesh resolution and viscous regularization on the wave profile, FD simulations were performed on a [100] single crystal shocked at $P^{-}=5 \mathrm{GPa}$. The resultant wave profiles are given in Figure 4 whereas computation times and computed dislocation densities in the shocked state are given in Table 5. Figure 4 illustrates that when an artificial viscosity is employed in conjunction with a mesh resolution that is too coarse to resolve the shock width predicted from the viscoplastic constitutive relations alone, the shock is smoothed. Consequently, Table 5 indicates that although computation times approach those associated with the SW method, the viscoplastic behavior, indicated by the total dislocation density in the shocked state, is altered due to the decrease in peak strain rate associated with the shock. When this damping occurs the viscoplastic behavior becomes highly mesh-dependent. Consequently, physical meaning associated with internal state variables that govern viscoplastic deformation and the ability to predict detailed wave profile evolution are lost in the FD approach with nonzero artificial viscosity.

Although the analysis in this section used a fixed set of shock viscosity parameters (recall $a_{1}=0.05$ and $a_{2}=2.0$ ), without significantly decreasing these parameters

Table 4 Total computation times on a single $2.67 \mathrm{GHz}$ Intel Xeon X5650 processor

\begin{tabular}{llll}
\hline Simulation & \multicolumn{2}{l}{ Machine time (s) } & Speedup factor \\
\cline { 2 - 3 } & SW & FD & \\
\hline 5 GPa [100] & 32.38 & $6.69 \times 10^{4}$ & 2006 \\
5 GPa low symmetry & 58.24 & $1.69 \times 10^{5}$ & 2902 \\
25 GPa [100] & 15.99 & $1.27 \times 10^{5}$ & 7942 \\
25 GPa low symmetry & 70.43 & $1.28 \times 10^{5}$ & 1817 \\
\hline
\end{tabular}




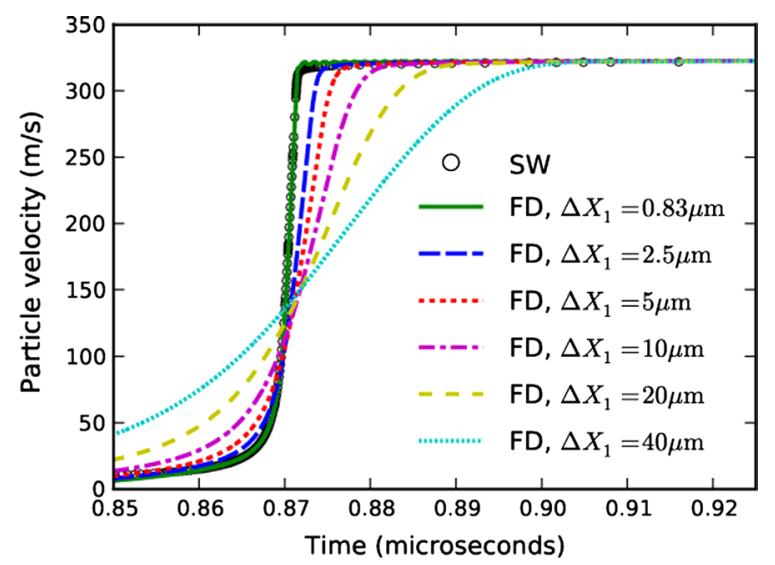

Figure 4 Longitudinal velocity $v_{1}$ computed using FD method with varying mesh resolution compared with SW method predictions ( $P^{-}=5 \mathrm{GPa}$ ). All FD simulations used an artificial viscosity, (28), except $\Delta X_{1}=0.83 \mu \mathrm{m}$.

Table 5 Computational time and total dislocation density for [100] shock at $P^{-}=5 \mathrm{GPa}$ computed using the FD method with varying mesh resolutions

\begin{tabular}{lll}
\hline Mesh resolution $\boldsymbol{\Delta} \boldsymbol{X}_{\mathbf{1}}(\boldsymbol{\mu} \mathbf{m})$ & Machine time $(\mathbf{s})$ & Total dislocation density $\left(\boldsymbol{\mu \mathbf { m } ^ { - 2 } )}\right.$ \\
\hline 0.83 & $6.69 \times 10^{4}$ & 270 \\
2.5 & $8.06 \times 10^{3}$ & 212 \\
5 & $2.08 \times 10^{3}$ & 192 \\
10 & 562 & 176 \\
20 & 159 & 177 \\
40 & 51.5 & 172 \\
\hline
\end{tabular}

a similar conclusion is reached. In cases where mesh resolution is not fine enough to resolve the viscoplastic shock behavior, the shock is smoothed across three to five elements by the quadratic component of the viscosity. The quadratic viscous pressure is proportional to the square of the jump in velocity, so unless $a_{2}$ is altered by orders of magnitude, the viscous pressure is determined by the magnitude of the velocity jump, and not by the relative magnitude of $a_{2}$.

\section{Comparison of numerical and analytical solutions}

Aspects of numerical solutions obtained using the FD approach of "Finite difference model" and the SW approach of "Steady wave model" are now compared with available results from the analytical approach outlined in "Analytical model". Specifically, the analysis is applied towards pure $\mathrm{Al}$ single crystals shocked along [100] to stresses of $P^{-}=5 \mathrm{GPa}$ and $P^{-}=25 \mathrm{GPa}$, the former corresponding to a weak plastic shock with an elastic precursor and the latter to a single overdriven plastic wave. Tables 6 and 7 list outcomes of the computation/analysis-volume ratio, resolved shear stress, cumulative plastic strain, total temperature rise, particle velocity, and shock velocity-for shocks of strength 5 and $25 \mathrm{GPa}$, respectively, obtained from the FD method, SW method, and analytical solution. 
Table 6 Shocked state of AI [100] $\left(P^{-}=5 \mathrm{GPa}\right)$

\begin{tabular}{lcccc}
\hline Variable (units) & FD & SW & Analytical & Birch-Murnaghan EOS \\
\hline$\lambda^{-}$ & 0.944 & 0.944 & 0.945 & 0.944 \\
$\tau^{-}(\mathrm{MPa})$ & 56.7 & 55.8 & 120.4 & 0 \\
$\left(\bar{\epsilon}_{e f f}^{P}\right)^{-}$ & 0.037 & 0.037 & 0.034 & 0 \\
$(\Delta \theta)^{-}(\mathrm{K})$ & 40.5 & 39.2 & 42.3 & 38.36 \\
$v^{-}(\mathrm{km} / \mathrm{s})$ & 0.323 & 0.323 & 0.319 & 0.321 \\
$D(\mathrm{~km} / \mathrm{s})$ & 5.739 & 5.717 & 5.798 & 5.771 \\
\hline
\end{tabular}

Table 7 Shocked state of Al [100] $\left(P^{-}=25 \mathrm{GPa}\right)$

\begin{tabular}{lcccc}
\hline Variable (units) & FD & SW & Analytical & Birch-Murnaghan EOS \\
\hline$\lambda^{-}$ & 0.810 & 0.805 & 0.805 & 0.816 \\
$\tau^{-}(\mathrm{GPa})$ & 1.023 & 1.047 & 0.417 & 0 \\
$\left(\bar{\epsilon}_{\text {eff }}^{P}\right)^{-}$ & 0.113 & 0.110 & 0.131 & 0 \\
$(\Delta \theta)^{-}(\mathrm{K})$ & 256.1 & 269.1 & 250.8 & 126.56 \\
$v^{-}(\mathrm{km} / \mathrm{s})$ & 1.324 & 1.341 & 1.343 & 1.303 \\
$D(\mathrm{~km} / \mathrm{s})$ & 7.000 & 6.882 & 6.879 & 7.105 \\
\hline
\end{tabular}

The precursor velocity calculated from the analytical nonlinear elastic solution [16, 29] is $6.28 \mathrm{~km} / \mathrm{s}$, which is exceeded by $D$ for the overdriven shock at $P^{-}=25 \mathrm{GPa}$. Also shown for comparison are hydrodynamic predictions obtained using the Birch-Murnaghan pressure-volume equation of state (EOS) [33], with compressibility properties of Al from the literature [34]. Temperature rise in the EOS was calculated assuming compression is isentropic and internal energy is first order in entropy. The hydrodynamic approximation, which by construction omits shear/deviatoric stress components, is often used as a simple model for shocks in materials whose strength is low relative to shock pressure.

The analytical solutions are obtained nearly instantaneously, in contrast to the more computationally intensive numerical methods. However, the analytical solutions only apply for symmetric orientations for which a single slip variable $\gamma$ suffices (e.g., [100] for FCC crystals). While the yield condition used in the analytical solution benefits from extreme simplicity, explicit rate and temperature effects on flow stress are ignored. Furthermore, while only a single fitting parameter $(H)$ is required, rather than an extensive list as in Table 2, $H$ must still be prescribed via comparison with shear strength data from experiments or other more physically descriptive model output. Here, following the latter approach, $H$ has been calibrated such that cumulative plastic deformation

$$
\begin{aligned}
\bar{\epsilon}_{e f f}^{P} & =\sqrt{\frac{2}{3}} \int\left(\boldsymbol{L}_{\text {sym }}^{P}: \boldsymbol{L}_{\text {sym }}^{P}\right)^{1 / 2} \mathrm{~d} t=\sqrt{\frac{2}{3}} \int\left(\boldsymbol{D}^{P}: \boldsymbol{D}^{P}\right)^{1 / 2} \mathrm{~d} t \\
& =\gamma \sqrt{\frac{2}{3}\left[\sum_{i=1}^{8}\left(\boldsymbol{s}^{i} \otimes \boldsymbol{m}^{i}\right)_{\text {sym }}\right]:\left[\sum_{j=1}^{8}\left(\boldsymbol{s}^{j} \otimes \boldsymbol{m}^{j}\right)_{\text {sym }}\right]}
\end{aligned}
$$

predicted by the analysis for 5 and $25 \mathrm{GPa}$ shocks is in relatively close agreement (within $\approx 20 \%$ error) with that predicted by SW and FD models, as is evident from Tables 6 and 7. Results for all end state variables and shock velocity are nearly equal for FD and 
SW simulations. Because these or very similar values have already been compared with experimental data $[18,19,35-39]$ indicative of viscoplastic relaxation rates (e.g., strength, precursor decay, wave profiles in single crystals and polycrystals) in previous publications $[6,10]$, these numerical results are deemed physically accurate. Results for volumetric compression ratio, adiabatic temperature rise, particle velocity, and shock velocity obtained from the analytical solution are also very close to corresponding numerical results, and effective plastic strain is reasonably close as noted already, although it is reiterated that the hardening parameter $\mathrm{H}$ entering the analytical method is obtained by fitting plastic deformation to the FD results. The only major discrepancy between analytical and numerical solutions is slip system-level shear strength in the shocked state $\left(\tau^{-}\right)$, which appears to be over-predicted by the analytical solution for the 5 GPa shock and under-predicted for the 25 GPa shock. While it would be possible to more closely duplicate results of the numerical methods by using a more complex (i.e., nonlinear) hardening model than that prescribed in (43), such an approach would also suffer from requiring additional fitting of more parameter(s), detracting from the simplicity of the analytical approach. Similarly, using a simple viscoplastic model wherein the dislocation density and velocity are prescribed functions of the accumulated shear strain and shear stress, respectively, may produce results that are simpler to replicate using the analytical model [5]. However, such a model is unable to describe both the weak and strong shock loading regimes [9]. Previous isotropic representations [3, 7-9] are also unable to account for anisotropy of single crystals and textured polycrystals that are addressed by the present fully anisotropic theory.

Because the Birch-Murnaghan EOS assumes a spherical stress state, shear stress and plastic deformation are unresolved in Tables 6 and 7, and temperature rise is under-predicted since there is no contribution to dissipation from plastic slip. The EOS does, however, predict reasonably accurate values of relative volume, particle velocity, and shock velocity.

\section{Conclusion}

Analytical, FD, and SW numerical solutions have been compared for shock loading of single crystals using identical thermoelastic frameworks, but with rate- and temperatureindependent shear strength constitutive relations in the analytical approach, and rate- and temperature-dependent shear strength constitutive relations in the latter two methods. Scenarios exist in which each of these methods is most appropriate. These method comparisons have not been published in previous papers which have focused on each model and its results in isolation.

Given a material for which there are limited strength data and incomplete understanding of physical mechanisms governing dissipation during shock loading, the analytical approach provides rapid shock response characterization based on thermoelastic properties, which are often available in literature, and a simple empirical hardening relation. In this case wave profile information predicted by the SW and FD methods would be largely speculative unless physical or experimental insights could be used to suggest more realistic, and presumably more complex representations of dissipation mechanisms and their relation to material strength. 
On the other hand if there is data that quantifies the spatio-temporal of the velocity profile, or if rate-dependent micromechanical mechanisms that govern the viscoplastic material response are well characterized, the SW or FD methods may be more appropriate. In particular, the SW and FD methods can be used to predict the steady shock structure as well as give information regarding evolution of internal state variables and the thermodynamic state prior to, within, and after the shock. Due to its computational efficiency, the SW method is especially useful for developing constitutive equations prior to their implementation in FD frameworks. Additionally, in "Computational efficiency" it was shown that predicted rate-dependent behavior may be unphysically altered in FD simulations due to viscous damping effects unless a sufficiently fine mesh resolution is employed, whereas viscous damping is not required in the SW method. Only the FD method is capable of quantifying transient aspects of evolving shock waves, which is necessary to model spatio-temporal shock wave evolution data such as elastic precursor decay.

All three methods can be used to model highly symmetric single crystal orientations subjected to shock loading, but only the FD method can be used to capture quasi-longitudinal and quasi-transverse waves that arise in low symmetry crystal orientations. For weak shock loading in $\mathrm{Al}$, approximating deformation as uniaxial was shown to be reasonable. Therefore, the SW method should be preferred due to its computational efficiency and lack of artificial viscosity. For strong shocks, however, the response of low symmetry crystal orientations was poorly captured using the SW method. Therefore, when modeling strong shocks for low symmetry crystal orientations relative to the loading direction, the finite-difference method should be employed.

These conclusions can be extended to other cubic metals with similar elastic anisotropy. However, additional investigations are required before generalizing these conclusions to materials that exhibit significantly higher elastic anisotropy or materials with lower crystal symmetry.

Authors' contributions

JTL performed finite difference and steady wave simulations. JDC derived and calculated the analytical shock response. RAA and DLM helped create, derive, and evaluate the viscoplastic formulation in conjunction with JTL. All authors read and approved the final manuscript.

Author details

${ }^{1}$ Impact Physics Branch, US Army Research Laboratory, Aberdeen Proving Ground, MD 21005-5066, USA. ${ }^{2}$ Materials Modeling and Simulation Group, Lawrence Livermore National Laboratory, Livermore, CA 94550, USA. ${ }^{3}$ Woodruff School of Mechanical Engineering, Georgia Institute of Technology, Atlanta, GA 30332-0405, USA. ${ }^{4}$ School of Materials Science and Engineering, Georgia Institute of Technology, Atlanta, GA 30332-0405, USA.

\section{Acknowledgements}

The authors would like to thank Dr. Richard Becker for stimulating discussions concerning implementation of the FD method. This work was performed, in part (RAA), under the auspicies of the US Department of Energy by Lawrence Livermore National Laboratory under contract DE-AC52-07NA27344 (LLNL-JRNL-663743). DLM is grateful for the support of the Carter N. Paden, Jr. Distinguished Chair in Metals Processing.

Compliance with ethical guidelines

Competing interests

The authors declare that they have no competing interests.

Received: 20 January 2015 Accepted: 12 June 2015

Published online: 10 July 2015 


\section{References}

1. McQueen RG, Marsh SP, Taylor JW, Fritz JN, Carter WJ (1970) The equation of state of solids from shock wave studies. In: Kinslow R (ed) High velocity impact phenomena. Academic Press, New York, pp 293-417

2. Herrmann W, Hicks DL, Young EG (1971) Attenuation of elastic-plastic stress waves. In: Burke J, Weiss V (eds) Shock waves and the mechanical properties of solids. Syracuse University Press, New York, pp 23-63

3. Clifton R (1971) On the analysis of elastic visco-plastic waves of finite uniaxial strain. In: Burke J, Weiss V (eds) Shock waves and the mechanical properties of solids. Syracuse University Press, New York, pp 73-116

4. Johnson JN (1972) Calculation of plane-wave propagation in anisotropic elastic-plastic solids. J Appl Phys 43:2074-2082

5. Winey JM, Gupta YM (2006) Nonlinear anisotropic description for the thermomechanical response of shocked single crystals: inelastic deformation. J Appl Phys 99:023510

6. Lloyd JT, Clayton JD, Becker R, McDowell DL (2014) Simulation of shock wave propagation in single crystal and polycrystalline aluminum. Int J Plast 60:118-144

7. Molinari A, Ravichandran G (2004) Fundamental structure of steady plastic shock waves in metals. J Appl Phys 95:1718-1732

8. Austin RA, McDowell DL (2011) A dislocation-based constitutive model for viscoplastic deformation of fcc metals at very high strain rates. Int J Plast 27:1-24

9. Austin RA, McDowell DL (2012) Parameterization of a rate-dependent model of shock-induced plasticity for copper, nickel, and aluminum. Int J Plast 32-33:134-154

10. Lloyd JT, Clayton JD, Austin RA, McDowell DL (2014) Plane wave simulation of elastic-viscoplastic single crystals. J Mech Phys Solids 69:14-32

11. Lloyd JT, Clayton JD, Austin RA, McDowell DL (2014) Modeling single-crystal microstructure evolution due to shock loading. J Phys Conf Ser 500:112040

12. Germain P, Lee EH (1973) On shock waves in elastic-plastic solids. J Mech Phys Solids 21:359-382

13. Johnson JN (1974) Wave velocities in shock-compressed cubic and hexagonal single crystals above the elastic limit. J Phys Chem Solids 35:609-616

14. Perrin G, Delannoy-Coutris M (1983) Analysis of plane elastic-plastic shock-waves from the fourth-order anharmonic theory. Mech Mater 2:139-153

15. Clayton JD (2014) Analysis of shock compression of strong single crystals with logarithmic thermoelastic-plastic theory. Int J Eng Sci 79:1-20

16. Clayton JD (2013) Nonlinear Eulerian thermoelasticity for anisotropic crystals. J Mech Phys Solids 61:1983-2014

17. Thomas JF (1968) Third-order elastic constants of aluminum. Phys Rev 175:955-962

18. Huang H, Asay JR (2006) Reshock response of shocked aluminum. J Appl Phys 100:043514

19. Turneare SJ, Gupta YM (2009) Real time synchrotron X-ray diffraction measurements to determine material strength of shocked single crystals following compression and release. J Appl Phys 106:033513

20. Clayton JD (2009) A continuum description of nonlinear elasticity, slip and twinning, with application to sapphire. Proc R Soc Lond A 465:307-334

21. McGlaun JM, Thompson SL, Elrick MG (1990) CTH: a three-dimensional shock wave physics code. Int J Impact Eng 10(1):351-360

22. Lloyd JT (2014) Microstructure-sensitive simulation of shock loading in metals. Ph.D. thesis, Georgia Institute of Technology, Atlanta, GA

23. Clayton JD (2011) Nonlinear mechanics of crystals. Springer, Dordrecht

24. Luscher DJ, Bronkhorst CA, Alleman CN, Addessio FL (2013) A model for finite-deformation nonlinear thermomechanical response of single crystal copper under shock conditions. J Mech Phys Solids 61:1877-1894

25. Clayton JD (2005) Dynamic plasticity and fracture in high density polycrystals: constitutive modeling and numerical simulation. J Mech Phys Solids 53:261-301

26. Becker R (2004) Effects of crystal plasticity on materials loaded at high pressures and strain rates. Int J Plast 20:1983-2006

27. Farren WS, Taylor GI (1925) The heat developed during plastic extension of metals. Proc R Soc Lond A 107:422-451

28. Wilkins ML (1980) Use of artificial viscosity in multidimensional fluid dynamic calculations. J Comput Phys 36(3):281-303

29. Thurston RN (1974) Waves in solids. In: Truesdell C (ed) Handbuch der Physik VIA/4. Springer, Berlin, pp 109-308

30. Waterman PC (1959) Orientation dependence of elastic waves in single crystals. Phys Rev 113(5):1240-1253

31. Winey JM, Gupta YM (2004) Nonlinear anisotropic description for shocked single crystals: thermoelastic response and pure mode wave propagation. J Appl Phys 96:1993-1999

32. Winey JM, Gupta YM, Hare DE (2001) R-axis sound speed and elastic properties of sapphire single crystals. J Appl Phys 90:3109-3111

33. Birch F (1947) Finite elastic strain of cubic crystals. Phys Rev 71(11):809-824

34. Vinet P, Rose JH, Ferrante J, Smith JR (1989) Universal features of the equation of state of solids. J Phys Condens Matter 1(11):1941-1963

35. Huang H, Asay JR (2007) Reshock and release response of aluminum single crystal. J Appl Phys 101(6):063550

36. Smith RF, Eggert JH, Jankowski A, Celliers PM, Edwards MJ, Gupta YM et al (2007) Stiff response of aluminum under ultrafast shockless compression to 110 gpa. Phys Rev Lett 98(6):065701

37. Gupta YM, Winey JM, Trivedi PB, LaLone BM, Smith RF, Eggert JH et al (2009) Large elastic wave amplitude and attenuation in shocked pure aluminum. J Appl Phys 105(3):036107

38. Turneaure SJ, Gupta YM (2011) Material strength determination in the shock compressed state using X-ray diffraction measurements. J Appl Phys 109(12):123510

39. Crowhurst JC, Armstrong MR, Knight KB, Zaug JM, Behymer EM (2011) Invariance of the dissipative action at ultrahigh strain rates above the strong shock threshold. Phys Rev Lett 107(14):144302 